\title{
TEMPO-FIT HEART RATE APP: USING HEART RATE SONIFICATION AS EXERCISE PERFORMANCE FEEDBACK
}

\author{
Steven Landry, Yuangjing Sun, Darnishia Slade, and Myounghoon Jeon \\ Michigan Technological University, \\ Mind Music Machine Lab, \\ 1400 Townsend Drive, Houghton, MI 49931 \\ \{sglandry, ysun4, dslade, mjeon\}@mtu.edu
}

\begin{abstract}
Physical inactivity is a worldwide issue causing a variety of health problems. Exploring novel ways to encourage people to engage in physical activity is a topic at the forefront of research for countless stakeholders. Based upon a review of the literature, a pilot study, and exit interviews, we propose an app prototype that utilizes music tempo manipulation to guide users into a target heart rate zone during an exercise session. A study was conducted with 26 participants in a fifteenminute cycling session using different sonification mappings and combinations of audiovisual feedback based on the user's current heart rate. Results suggest manipulating the playback speed of music in real time based on heart rate zone departures can be an effective motivational tool for increasing or decreasing activity levels of the listener. Participants vastly preferred prescriptive sonifications mappings over descriptive mappings, due to people's natural inclination to follow the tempo of music.
\end{abstract}

\section{INTRODUCTION}

Physical inactivity is a worldwide issue, causing health problems such as obesity, high blood pressure, diabetes, and other psychosocial problems [1]. Finding new and effective ways to motivate and guide users in their exercise sessions could help solve this epidemic of inacitvity. Music is known to distract listeners from the monotony of exercise, and music tempo has a direct link to both percieved and acutal physical output [2] of a work out session. This paper explores the viability of using music tempo manipulation as a means to guide and motivate users to exercise more effectively. The mission of the Tempo-Fit Heart Rate App is to offer users a simple, easy to use, effective, and motivating approach to improving their physical activity and achieving target health goals through the use of musical tempo feedback.

Previous studies have examined the viability of sonifiying physiological data to guide runners into a predefined optimal heart range. For instance, Wärnegård developed and tested an app that provided auditory warnings (Earcons) when the user's heart rate fell outside of the predefined optimal range. This sonification strategy helped users maintain a consistent heart rate inside the optimal range

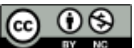
Attribution - Non Commercial 4.0 International License.

The full terms of the License are available at

http://creativecommons.org/licenses/by-nc/4.0/ compared to a control condition [3]. Instead of discrete Earcons, the proposed Tempo-Fit app modifies the playback speed (tempo) of the user's music to either reflect current heart rate (descriptive, or "what you are doing") or guide the user (perspective, or "what you should be doing") back to the optimal zone. Using this type of feedback system allows listeners to continuously monitor their changing HR while also providing more interesting and motivating auditory cues than simple Earcons.

Auditory research has shown time and time again the relationship between music tempo preference and exercise intensity [4]. Multiple studies have observed that when exercising, participants vastly prefer to listen to music with medium to high tempos ( $<120 \mathrm{bpm})$. Even for low intensity

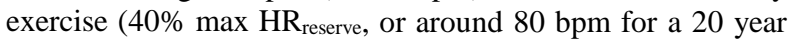
old), participants showed a significant preference for both medium (120bpm) and high (140bpm) tempo music over slow tempos (80bpm). The same preference was found for higher intensity exercises $\left(75 \%\right.$ max $\mathrm{HR}_{\text {reserve, }}$ or around $150 \mathrm{bpm}$ for a 20 year old), suggesting the relationship between HR during exercise and music tempo preference is not perfectly linear. Multiple exercise motivational applications (such as MusicalHeart, RockMyRun, Mptrain, etc.) analyze heart or step rate of the exerciser to suggest songs from a database with matching BPM’s [5, 6, 7].

The Tempo-Fit heart rate application presented in this paper takes a different approach to using the BPM of music for exercise motivation. The music tempo (or in this case, speed of playback) is manipulated in real time to give cues to the listener's current activity level. Since it is not necessary to match the exact music BPM to heart rate, and there already exists a general preference to medium to high music BPM's regardless of exercise intensity level [4], users are allowed to select their own music. The application speeds up or slows down the playback speed of the current song as an auditory cue that the user's heart rate has dropped below or above the recommended (or preset) HR range. Playback of the audio file is temporarilly manipulated to either $125 \%$ or $75 \%$ speed. This manipulation gradually increases or decreases over five seconds, emphasizing the change in tempo, as opposed to the actual BPM of the song. Once the user's HR returns to the desired range, the playback speed of the audio file immediately returns to $100 \%$ (normal) speed. Our hypothesis is that the gradual change in tempo will be a more effective motivational feedback cue than the actual tempo of the music at any point in time. To test this hypothesis, and to gauge preference for either prescriptive (what you should be doing) or descriptive (what you are doing) sonification mapping, we 
invited participants to try out our system during a fifteen minute exercise session on a cycling machine.

\section{METHODS}

\subsection{Participants}

All participants $\left(N=26,4\right.$ females, $M_{\text {age }}=20.1, S D_{\text {age }}=1.3$, age range: 18-22 years) were recruited from the local university undergraduate participant pool. Each participant was compensated with two psychology course credit points for an hour long study. The majority of participants (20/26) rated themselves as fairly athletic and reported going to the gym or working out semi-regularly (at least once per week). All participants were screened for medical conditions associated with increased risks of complications in relation to physical exertion. No other demographic information was collected.

\subsection{Stimuli and equipment}

Figure 1 depicts the laboratory setting where the cycling session took place. A 20 inch computer monitor was positioned three feet in front of the participant for television viewing. A smartphone (Galaxy S3) was placed on a table approximately two feet away from the participant. The smartphone app displayed a visual HR (approximately two squared centimeters in size) slightly outside of the participant's field of view, forcing the participant to turn their head away from the television monitor to visually check their heart rate readout from the smartphone. A Monark 818E cycling machine (with participant-chosen resistance weight; normally between .25 and $2.0 \mathrm{KG}$ ) was used as the exercise equipment. The music stimuli were played from computer speakers positioned 3 feet away from the participant at a volume level averaging around $80 \mathrm{~dB}$. All participants heard the same four songs (see Table 1) in the same order approximately twice during the exercise session. All songs fit into the music genre of dance music, varying from 117-130 BPM.

Table 1: Track titles and BPM of each song used for auditory feedback

\begin{tabular}{|l|l|}
\hline Artist - Song title & BPM \\
\hline \hline Adam Lambert - For Your Entertainment & 130 \\
\hline \hline Daft Punk - One More Time & 123 \\
\hline \hline Casio Kids - Fot I Hos & 128 \\
\hline \hline Justin Timberlake - Sexyback & 117 \\
\hline
\end{tabular}

\subsection{Conditions}

A between-subjects design was implemented where participants were randomly assigned to one of the following 4 conditions:

Control - No tempo manipulation (music played at normal speed regardless of participant's HR). HR was visually displayed on the smart phone (updated once every five seconds) positioned next to the participant.

Prescriptive - "What you should be doing" mapping: Music tempo is gradually increased (over five seconds) to $125 \%$ speed when the participant's HR was below the target range. Music tempo is gradually decreased (over five seconds) to $75 \%$ speed when the participant's HR was above the target range. Once the participant's HR returned to the target range, the music tempo immediately jumped back to $100 \%$ (normal) speed. Visual feedback was also provided in the same manner as the control group.

Descriptive - "What you are doing" mapping: Opposite to the prescriptive condition; the music tempo increased to $125 \%$ speed when the participant's HR was above the target range. Music tempo decreased to $75 \%$ when the participant's HR was below the target range. Music returned to $100 \%$ (normal) speed immediately when the participant's HR was within the target range. Visual feedback was provided in the same manner as the above conditions.

Music Only - Auditory only, no visual feedback: Auditory feedback was provided in the same manner as the prescriptive condition ("What you should be doing" mapping). No visual HR information was provided.

\subsection{Procedure}

Following the consent form and screening questionnaire, the participant equipped the Equivital vest [8] which sends the participant's HR data over Bluetooth to the smartphone app once every five seconds. The participant was then instructed to select a TV episode to watch from Netflix.com to watch on the computer monitor. The TV sound was muted and subtitles were enabled to more closely resemble a gym environment and to provide incentive to use the auditory display over the visual display. All but four participants chose comedic cartoon shows such as "Family Guy" or "Futurama".

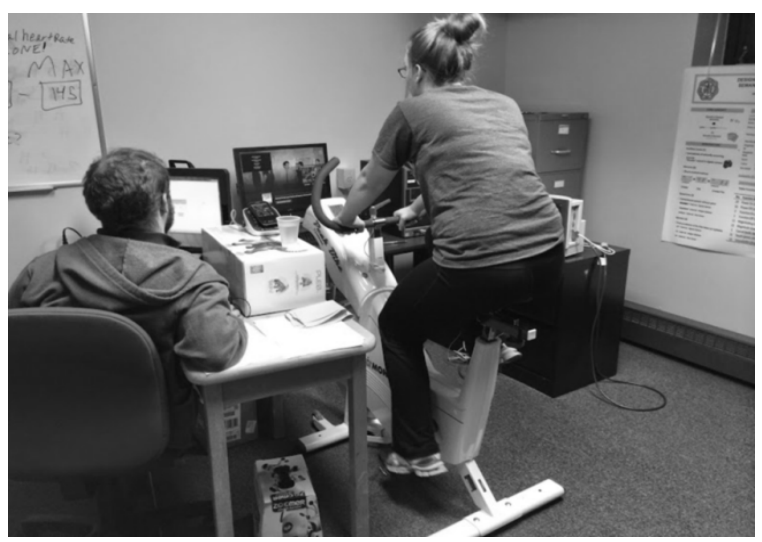

Figure 1: Actual setup of experiment, including: TV monitor for Netflix.com, smartphone (Galaxy S3), Monark 818E cycling machine. Music played from experimenter controlled PC speakers.

After selecting the show on Netflix, the target HR range was calculated for each participant using the formula: $\mathrm{min}=$ $\left[(220 \text {-age })^{*} .5\right]+5, \max =\left[(220 \text {-age })^{*} .6\right]-5$ [8]. Five was added to the minimum and subtracted from the maximum to decrease the target zone from a range of 20 to a range of 10 to prevent a ceiling effect on performance. The majority of the participants' personally calculated target heart rate ranges were around 125-135 BPM. The participant was then instructed on his or her group's particular sonification mapping.

After confirmation that the participant understood the instructions, he or she would begin pedaling on the Monark 818E cycling machine. Once the participant's HR was within 
the target range for 20 consecutive seconds, the experimenter would begin the Netflix TV show, the music, and a 15 minute timer. The experimenter manually adjusted the playback speed of the music in a "Wizard of Oz" fashion. Tempo manipulation was done via a custom Max/MSP patch. After the 15 minute cycling period, the experimenter conducted a semi-structured interview before releasing the participant to assess user preferences.

To operationalize performance of the different feedback strategies, the percentage of time the user's heart rate fell within the target range was calculated for each participant (heart rate samples within target range / total samples = percentage of time in target range).

\section{RESULTS \& DISCUSSION}

Each participant experienced one condition, and the percent of HR samples (1 HR sample every 5 seconds) was used as the dependent variable. From Figure 2 we interpreted no significant differences $(p>.05)$ between group performances due to the overlapping 95\% confidence intervals and extremely small sample sizes within groups. However, this suggests that the music only condition performed as well as all other conditions including visual feedback.

\section{Avg $\%$ time in range by condition}

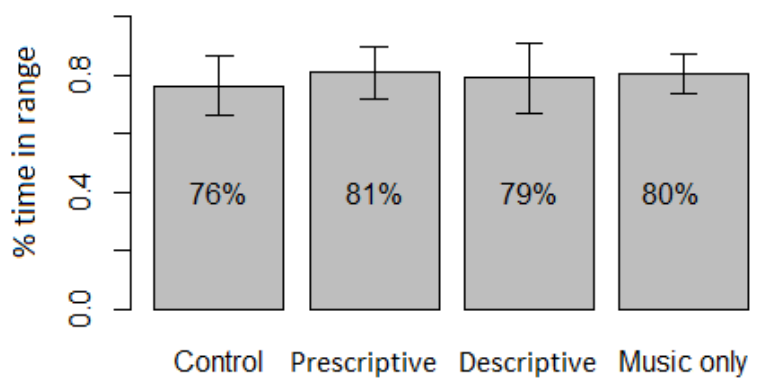

Figure 2: Average percent in range by condition. All error bars represent $95 \%$ confidence intervals.

All but three participants said they would use the app if available. Participants reported the level of activity of the cycling session to be equivalent to a brisk walk or light jog. All participants (except those in the control condition) considered the music helpful. Participants in prescriptive and descriptive conditions reported a strategy of watching TV for 20-30 seconds, checking the visual read out for 1-3 seconds, then back to the TV. Participants in the control condition compensated without auditory feedback by ignoring the TV show and allocating attention primarily on reading the small HR digit. Even with this compensation strategy, participants in the control condition stayed within the target range for $76 \%$ of the session (lowest of all four conditions) compared to $80 \%$ in the music only condition. Those participants in the descriptive condition often reported that the mapping of HR to tempo was not intuitive, or confusing. For instance, whenever a participant in this condition's HR increased above the target range, the tempo of the music would increase to $125 \%$. This increase in tempo was sometimes associated with an involuntary 3-5 BPM spike in the participant's HR even though the participant knew that an increase in the tempo of the music was used to suggest that they should decrease their activity level to return their HR to the target range. Figure 3 shows the sampled HR from a participant in the descriptive condition, and figure 4 shows the sampled HR from a participant in the prescriptive condition.

P\#2 Heart Rate (prescriptive conidition)

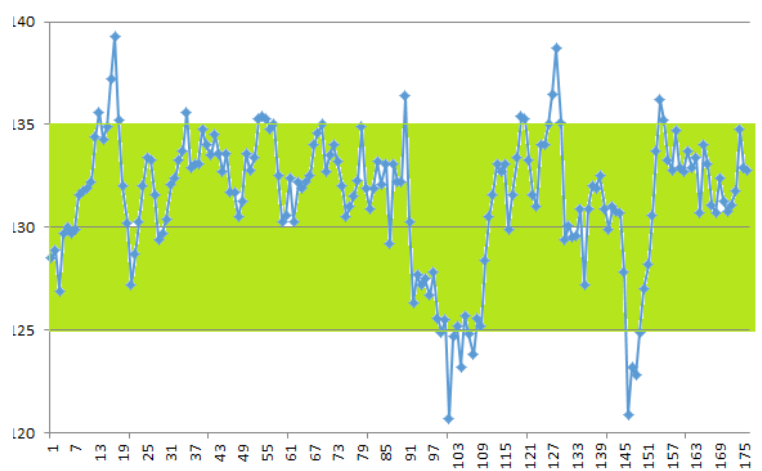

Figure 3: One participant's HR (bpm) over time. The shaded area represents the target HR range. The participant was in the prescriptive condition.

P\# 11 Heart Rate (descriptive condition)

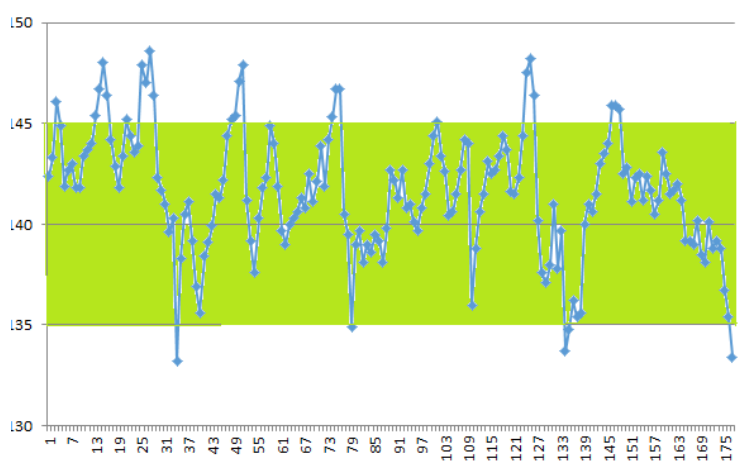

Figure 4: One participant's HR (bpm) over time. The shaded area represents the target HR range. The participant was in the descriptive condition.

Figure 3 shows that the participant easily manages to return back into the target range within 5-10 seconds or 1-2 HR samples in the prescriptive condition. Compare this to Figure 4 where each time the participant's (in the descriptive condition) HR exceeds the target range, there is an additional 3-5 bpm spike in HR, and it takes longer for that participant to return to the target range (on average, 3-4 HR samples or 15-20 seconds). To further analyze this trend, the average time per target zone departure was calculated for both above the target range. For each time a participant left the target HR zone, the number of samples was counted until the participant returned to the target zone. The sum of samples in each departure divided by the number of departures in a session gives an estimate of the average time spent per departure for that individual. 


\section{Avg time in an over departure}

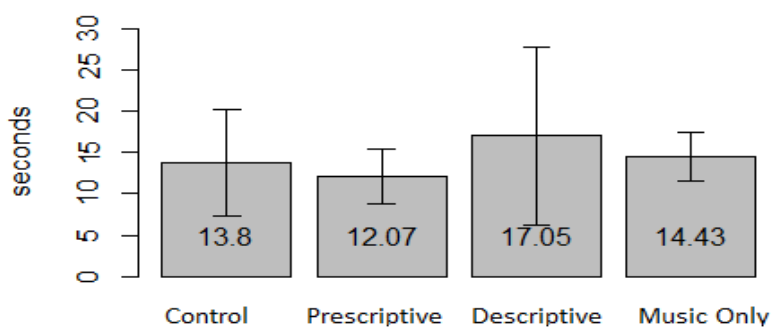

Figure 5: Average time spent in an “over departure” per condition.

Analyzing the data in this fashion gives an estimate of reaction time when exposed to different types of feedback. Reaction time in this sense is how quickly a participant reduces their heart rate back into the target range after exposure to a tempo manipulation. Again, the 95\% confidence intervals in each group are overlapping, suggesting no significant differences. However, there is a trend of participants in the descriptive condition needing an extra 5 seconds on average to bring their heart rate down to the target range compared to participants in the prescriptive condition. In other words, participants found the prescriptive sonification mapping technique to be more intuitive than the target matching descriptive approach. This lends further support to the idea that it is people's natural inclination to follow the rhythm of the music, as opposed to descriptive mapping where the tempo reflects the user's current HR.

\section{CONCLUSIONS}

The results of the study suggest two main points. The first is that the change in music tempo served an effective motivational element, perhaps more than the actual tempo of the songs we selected. The second conclusion is that prescriptive sonification mapping (what you should be doing) is more intuitive than the descriptive mapping (what you are doing). This is probably explained by people's natural inclination to synchronize their movements (or generally, their activity level) to the speed of the music, not the other way around. Subjective feedback from the semi structured interviews indicated that participants enjoyed the novelty of hearing familiar songs at unfamiliar speeds. Additionally, participants reported that they enjoyed the "game aspect" of controlling the playback speed of the music with their physical activity output.

The main limitation of this study is the small sample size (six per condition). Perhaps a significant difference could be found between conditions if more participants were included (if there truly is one to be found). Level of athleticism could have biased our results as a confounding variable that was not controlled for. The self-reported athletes in the study seemed to have an easier time controlling their heart rate (due to prior experience with target heart rate zones). Either way, a follow up study should be employed utilizing a within-subjects design with more participants, including a baseline condition with no heart rate feedback whatsoever to compare to.

Based on this small study, the use of music tempo manipulation as feedback on physical activity has shown to be as effective (and more enjoyable) than visual feedback. All 26 participants agreed that for guiding a user towards a target heart rate range, the mapping utilized in the prescribing condition was most appropriate. This type of auditory feedback would be most helpful for exercises such as running or cycling, where it may disrupt the user's flow to pull out a smartphone or watch and visually check for current heart rate. Some participants suggested that the opposite mapping (the type used in the describing condition) could be effective for achieving short bursts of high activity, as required for sprinting and weight lifting. If people's intuitive reaction to an increase in tempo is to increase physical output, perhaps this mapping could result in a positive feedback loop, where the sensor detects an increase in heart rate (associated with the start of a sprint or end of a lifting set), and the system increases the tempo of the music resulting in an extra burst of physical output by the user. Alternatively, the same mapping could create a negative feedback loop where a decrease in physical activity is required, such as yoga or meditation. Further research is needed to validate this type of sonification in these specific circumstances. For instance, a few participants reported that they listened to podcasts or audiobooks while exercising. A future usability study could use similar sonification mapping techniques applied to spoken word audio to see if it would have a similar effect on exercise performance.

A version of the Tempo-Fit Heart Rate application is in the process of being made for android smartphones using the libPD library for playback speed manipulation. It will communicate with a variety of popular wearable HR monitors that use the BluetoothHealth API (Polar, Garmin, etc.). Once completed, it will be uploaded to the Google Play market for free and its source code released on GitHub.

\section{REFERENCES}

[1] http://www.cdc.gov/physicalactivity/data/facts.html

[2] J. Edworthy and H. Waring, "The Effects of music tempo and loudness level on treadmill exercise," Ergonomics, 2006, pp. 1597-1610.

[3] H. Wärnegård, "Heart rate sonification-using sound to monitor heart beat when running," student thesis, 2012.

[4] C. Karageorghis, I. Costas, L. Jones, and D. Low. "Relationship between exercise heart rate and music tempo preference," Research Quarterly for Exercise and Sport, 77.2 (2006): 240-250.

[5] S. Nirjon, R. Shahriar, R. Dickerson, Q. Li, P. A. John, A. Stankovic, D. Hong, B. Zhang, X. Jiang, G. Shen, and F. Zhao. "Musicalheart: A hearty way of listening to music," Proceedings of the 10th ACM Conference on Embedded Network Sensor Systems. ACM, 2012.

[6] N. Oliver, and F. Flores-Mangas. "MPTrain: a mobile, music and physiology-based personal trainer," Proceedings of the 8th conference on Human-computer interaction with mobile devices and services. ACM, 2006.

[7] C. Bauer, and A. Kratschmar. "Designing a Musiccontrolled Running Application: a Sports Science and Psychological Perspective," Proceedings of the 33rd Annual ACM Conference Extended Abstracts on Human Factors in Computing Systems. ACM, 2015.

[8] http://www.equivital.co.uk/products/tnr

[9] http://www.acsm.org/access-publicinformation/articles/2012/01/13/the-heart-rate-debate 